\title{
DESARROLLO DE UN PROGRAMA COMPUTACIONAL ESPECIALIZADO PARA INGENIERÍA HIDRÁULICA E HIDROLOGICA "S-Hidro v 1.0"
}

\section{RESUMEN}

Presentamos un programa computacional codificado en MATLAB, el cual es una herramienta muy útil para la programación de cálculos para procesos hidráulicos e hidrológicos, Una de las principales ventajas que presenta este programa es su potencia y versatilidad en cuanto al manejo de archivos externos, es decir que se pueden importar archivos de Excel ( $\left.{ }^{\star} . x \mid s\right) 0$ archivos tipo texto $\left({ }^{\star}\right.$. txt); por tanto, facilita el manejo de archivos de datos y su procesamiento utilizando técnicas estadisticas desarrolladas y codificadas en este trabajo.

Palabras Clave: Análisis estadístico, datos hidrológicos, programas para hidrologia, programas MATLAB

\section{ABSTRACT}

We present this computing program encoded in matlab which is a useful tool to do calculation for the hydraulic and hydrological processing. One of the main advantages which this program presents is its power and versatility related to the manage of external files. It means we can import excel files ( $\left.{ }^{*} . x \mid s\right)$ or text files $\left({ }^{*} . t x t\right)$. Consequently, this facilitates the manage of the data files and their processing using statistical techniques developed and encoded in this paper.

Keywords: statistical analysis, hydrological data, programs for hydrology, matlab programs.

\section{INTRODUCCION}

La solución de muchos problemas en hidrologia requiere del uso de computadoras, las cuales simplifican los cálculos laboriosos. Sin embargo, para elaborar un programa de cómputo se requiere un amplio conocimiento del fenómeno, su correspondiente modelo matemático y la respectiva codificación en algún lenguaje de programación.

Los datos hidrológicos, frecuentemente requieren ser utilizados para el diseño de estructuras hidráulicas, ya sea con fines de utilización de previsión ante desastres naturales.

Dichos datos hidrológicos son normalmente de registros extensos y de naturaleza muy variable, por lo que antes de ser utilizados requieren pasar por un estricto control estadístico a lo que le denominamos análisis de homogeneidad o consistencia y luego el análisis de frecuencias hidrológicas, con lo cual quedan expeditos para ser modelados.

SHIDRO versión 1.0, es un programa de cómputo elaborado en MATLAB, con la finalidad de contar con una herramienta ágil y versátil para el procesamiento, análisis y modelación de datos hidrológicos. La elaboración de este programa se da en virtud a las dificultades que se presentan al momento de utilizar los datos históricos para la determinación de caudales de diseño u otros usos.

\section{DESARROLLOY RESULTADOS}

SHIDRO versión 1.0 es totalmente amigable para el usuario, se han diseñado un conjunto de pantallas que orientan al usuario paso a paso para lograr los objetivos del análisis estadístico, presenta menús desplegables o de cascada, que permiten importar el archivo de datos de interés y hacer el análisis estadístico que se requiera, desde la visualización en forma de gráfica hasta resultados en forma tabular, parámetros calculados y comparaciones estadísticas.

La pantalla principal de SHIDRO v $1.0 \mathrm{se}$ muestra a continuación, en la misma que se hace referencia a las bondades, alcances del programa presentado y los módulos desarrollados en la versión presentada.

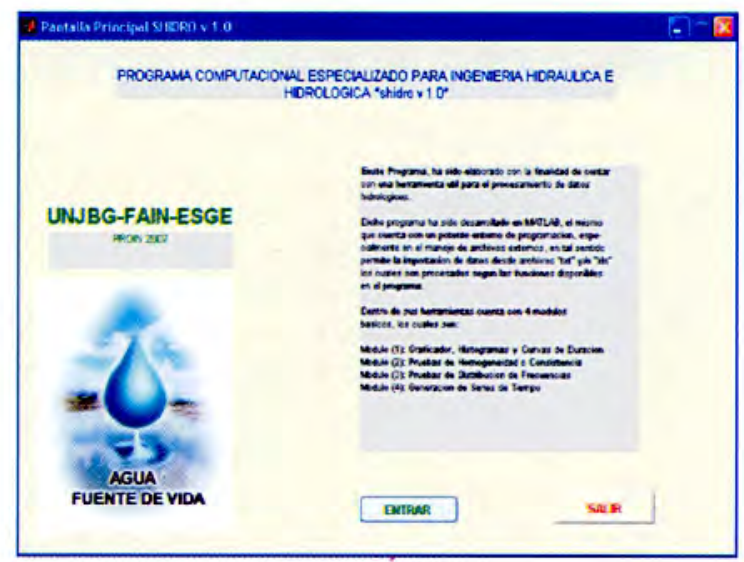


El programa presenta cuatro (04) módulos básicos los cuales se muestran en la siguiente pantalla y corresponde a: Modulo 1, Graficador, Histogramas y Curvas de Duración. El Módulo 2 , corresponde al programa que permite realizar las pruebas estadísticas de homogeneidad o consistencia, las cuales son muy útiles para verificar la homogeneidad o consistencia de la data hidrológica.

El Módulo 3 corresponde al programa para realizar el análisis de frecuencias hidrológicas, utilizando comparaciones estadísticas entre las distribuciones teóricas y la distribución de los datos primigenios. El módulo 4 permite generar series de tiempo, previa visualización del correlograma y datos aleatorios generados. EI programa también permite obtener archivos de salida en formato *.xls para su posterior tabulación y presentación de ser requerido

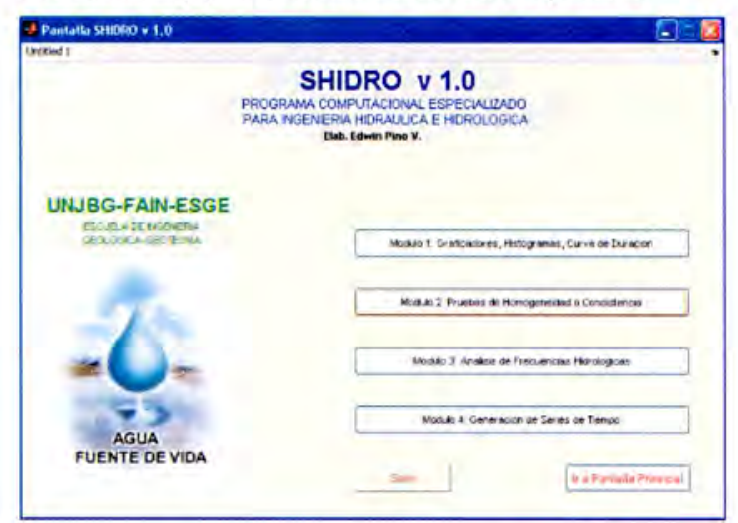

Módulo 1: Graficador, Histogramas y Curvas de Duración.

Es un módulo especialmente útil para el manejo previo o inicial de los datos hidrológicos, fácil de utilizar y con grandes resultados gráficos y tabulares para su interpretación. Las pantallas muestran algunas de las aplicaciones realizadas a la estación de descargas Calientes, sobre el río Caplina en la Región Tacna. En este caso de aplicación se realizó la importación de datos desde el menú de cascada y dando la ruta adecuada se importó el archivo de datos que pudo ser manipulado y procesado con todas las opciones que presenta el programa en este primer módulo.

Es posible obtener diferentes tipos de gráficos, curvas de duración para los datos promedio mensuales o para toda la serie histórica, además nos permite obtener el valor a diferentes niveles de persistencia según sea requerido. La siguiente pantalla muestra la curva de duración para toda la serie histórica de la estación de descargas Calientes.

En la siguiente pantalla se muestra un gráfico que presenta la distribución de descargas medias y los límites de confianza estadisticos.

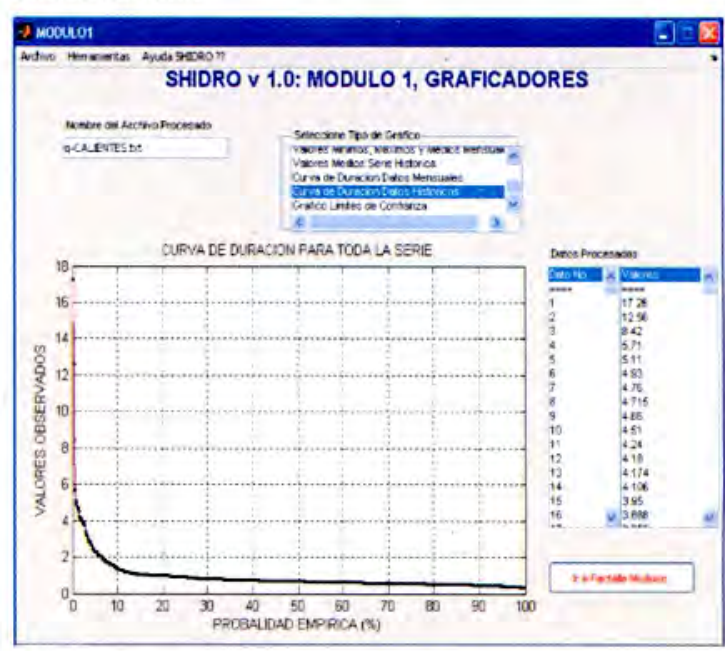

Módulo 2: Pruebas de Homogeneidad 0 Consistencia.

El test o prueba estadistica de homogeneidad presenta una hipótesis nula y una regla para aceptarla o rechazarla sobre la base de su probabilidad de ocurrencia. Si dicha probabilidad es pequeña, se concluirá que la serie es "no homogénea", si es grande la serie será "homogénea".

Para tal efecto se puede utilizar las pruebas paramétricas y no paramétricas. La prueba paramétrica se aplica si se puede especificar la distribución que sigue la hipótesis nula, de lo contrario si no es posible especificar la distribución que sigue la hipótesis nula las pruebas no paramétricas deben ser aplicadas.

Este módulo es muy completo en cuanto a pruebas de homogeneidad o consistencia; se han incluido las pruebas de homogeneidad paramétricas y no-paramétricas. Dentro de las pruebas No-Paramétricas se han desarrollado la prueba de las secuencias y la prueba de Helmert. En cuanto a las pruebas Paramétricas se han desarrollado y codificado en MATLAB las pruebas o test para media de una muestra, comparación de medias de dos muestras, test para variancia de una muestra, test para variancia de dos muestras y otras.

A continuación se muestran algunas pantallas de referencia, donde se puede observar la gran versatilidad del programa y forma de considerar la independencia de los datos analizados. Esta pantalla muestra la opción que corresponde a las pruebas estadisticas No-Paramétricas, es posible seleccionar el mes de análisis, se observa la distribución gráfica de los datos y se muestran los parámetros estadísticos calculados de la muestra y propios de la prueba. 


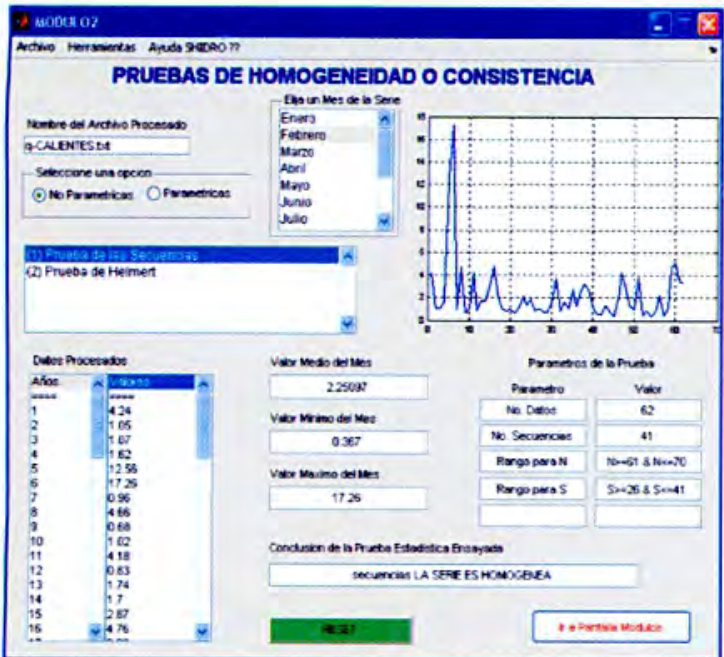

Módulo 3: Pruebas de Distribución de Frecuencias.

Módulo que permite probar estadisticamente diferentes distribuciones teóricas a los datos observados. El análisis de frecuencias es procedimiento para estimar la frecuencia de ocurrencia o probabilidad de ocurrencia de eventos pasados o futuros. EI análisis de frecuencias requiere que los datos sean homogéneos e independientes. La restricción de homogeneidad asegura que todas las observaciones provengan de la misma población. La restricción de independencia asegura que un evento hidrológico, tal como una gran tormenta aislada, no entre en el conjunto de datos más de una vez. En este módulo se han desarrollado códigos computacionales para pruebas de distribución de frecuencias a Distribución teóricas Normal, Distribución Weibull, además de los Test de Kolmogorov, Jarque Beta y Lillefords para distribución Normal. En este caso se puede importar también el archivo de datos, seleccionar el mes para el análisis, mostrar los valores en forma gráfica y tabular. Es posible visualizar el ploteo en papel de distribución normal, tal como se presenta a continuación y en el caso de los test estadísticos, se presentan los parámetros de la serie primigenia y los parámetros estadísticos respectivos.

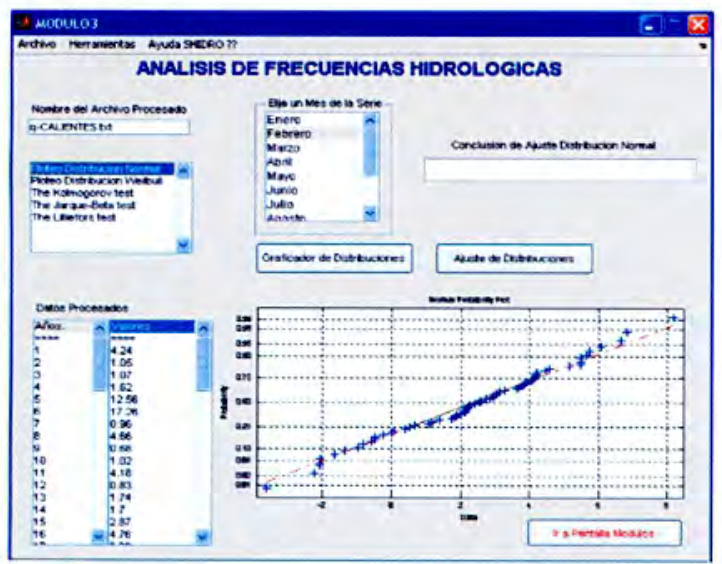

Pantalla que muestra la aplicación de un test de frecuencias para el ajuste a una distribución normal teórica. La conclusión estadística es de aceptación o rechazo de la hipótesis planteada

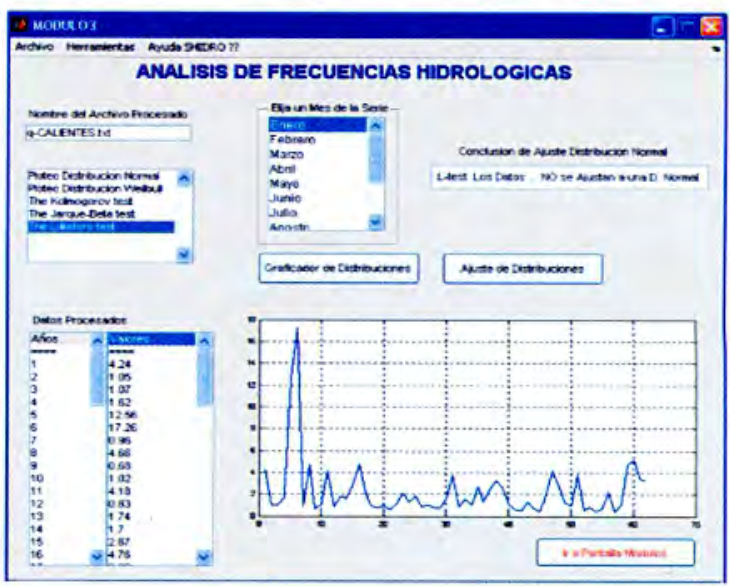

Módulo 4: Generación de Series de Tiempo, ha sido incluido con la finalidad de modelar las series hidrológicas y lograr la generación sintética de series de tiempo. Este módulo se encuentra en proceso de desarrollo, pero en esta versión es posible mostrar los correlogramas, generación de números aleatorios y generar series con el modelo de Monte Carlo. En realidad, la generación de series sintéticas bastante complejas, por lo que en esta versión se ha intentado empezar a resolver esta temática, habiéndose iniciado con el proceso inicial que corresponde a la generación de los correlogramas y la generación de números aleatorios que son muy importantes en la aplicación de los modelos estocásticos.

La siguiente pantalla muestra un correlograma calculado para los datos importados de la estación de descargas Calientes sobre el río Caplina, basado en la autocorrelación de su serie histórica.

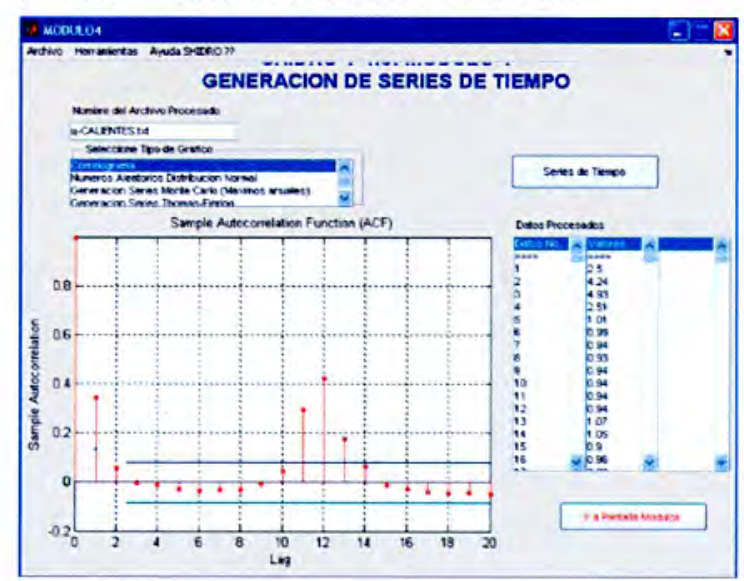

La siguiente pantalla nos muestra la comparación entre una serie observada y una generada según el modelo de Monte Carlo para la estación Calientes. Se debe mencionar que 
al picar sobre la misma opción que se denota en el recuadro "Seleccione Tipo de Gráfico", se puede obtener otra serie generada basada en la aleatoriedad de la componente probabilística del modelo. De esta versión podemos concluir que se lograron los objetivos trazados al inicio, que se referian a diseñar y elaborar un programa computacional que ayude en el tedioso trabajo de acondicionamiento y manejo de los datos hidrológicos. El programa es muy simple de utilizar y a su vez arroja resultados de mucha utilidad para el análisis y decisión del hidrólogo.

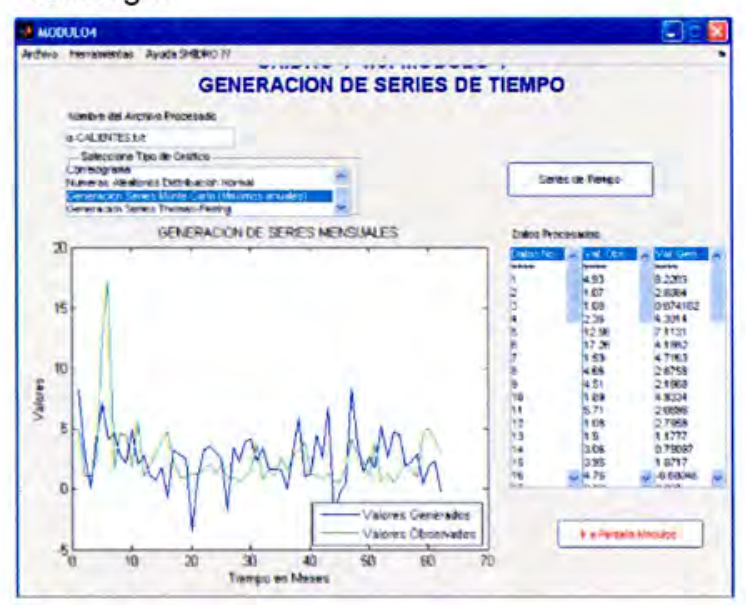

\section{CONCLUSIONES}

1.De esta versión podemos concluir que se lograron los objetivos trazados al inicio, que se referian a diseñar y elaborar un programa computacional que ayude en el tedioso trabajo de acondicionamiento y manejo de los datos hidrológicos.

2.El programa es muy simple de utilizar y a su vez arroja resultados de mucha utilidad para el análisis y decisión del hidrólogo.

3.Las series temporales pueden servir para predecir acontecimientos futuros basándose en ciertos comportamientos de determinadas variables. Si tenemos más observaciones que se puedan promediar, que es el orden de la media móvil, se obtienen tendencias más suaves. Este hecho no debe hacernos olvidar que aunque hemos mejorado la tendencia con el suavizado, por el contrario perdemos información sobre los valores iniciales y finales de la tendencia estimada.

\section{Finalmente podemos recomendar}

continuar con el desarrollo del cuarto módulo y agregar módulos relacionados con la simulación de embalses, hidráulica de canales abiertos y otros.

\section{RECOMENDACIONES}

Continuar con las investigaciones y trabajos referidos a la programación utilizando MATLAB y generar nuevas versiones actualizadas.

Incluir módulos con temas referidos a determinación de volúmenes de embalses, tránsitos de avenidas, hidráulica de canales.

Mejorar los módulos elaborados, especialmente es de pruebas estadísticas y generación de series de tiempo.

\section{BIBLIOGRAFÍA}

Linsley Kohler. Ed. McGraw Hill Book Co. Hidrología para Ingenieros.

\section{Ed. McGraw Hill Book Co.Handbook of Aplied Hydrology. Ven Te Chow Editor. \\ G. Monsalve Sáenz. Ed. Escuela Colombiana de Ingenieria. 1995. Hidrología en la Ingeniería.}

David R. Hidrologia Aplicada. Ven Te Chow Maidment, Larry. Mays. Mc.Graw Hill. 1994.

Métodos Estadísticos en Hidrologia. Mejía Marcacuzco Abel. La Molina 1994.

Separatas del Curso de Hidrología. Edwin Pino Vargas 2003.

Http://www.monografias.com/trabajos30/series -de-iempo/series-de-tiempo.shtml.

Www.mathworks.com/products/matlab

Www.math.utah.edu/lab/ms/matlab/matlab.ht $\mathrm{ml}$.

www.math.ufl.edu/help/matlab-tutorial. 